\title{
Evaluation von Stressprävention und Stressbewältigung mittels epigenetischer Marker
}

\author{
Martin Stoffel $^{a} \quad$ Elena Gardini $^{b, c} \quad$ Johannes C. Ehrenthal ${ }^{a} \quad$ Elvira Abbruzzese $^{b}$ \\ Beate Ditzen ${ }^{a}$ \\ anstitut für Medizinische Psychologie, Zentrum für Psychosoziale Medizin, Universitätsklinikum Heidelberg, \\ Heidelberg, Deutschland; ' ${ }^{b}$ Klinische Psychologie und Psychotherapie, Psychologisches Institut, Universität Zürich, \\ Zürich, Schweiz; ' ${ }^{C}$ nniversity Research Priority Program (URPP) Dynamics of Healthy Aging, Universität Zürich, \\ Zürich, Schweiz
}

Schlüsselwörter

Epigenetik · Methylierung · Stressbewältigung ·

Stressprävention

\section{Zusammenfassung}

Chronischer oder traumatischer Stress kann psychische Störungen und körperliche Erkrankungen begünstigen oder sogar verursachen. Ergebnisse aus der Grundlagenforschung zeigen, dass Stress zu einer Dysregulation wichtiger körperlicher Stresssysteme führen kann, welche diese Prozesse vermutlich vermitteln. Hierauf aufbauend wurden Interventionen zur Prävention oder zum Umgang mit Stress entwickelt, deren Wirkung mittels verschiedener Indikatoren dieser Stresssysteme evaluiert werden kann. In den letzten Jahren zeigten Ergebnisse aus Tier- und Humanstudien, dass sich Stress auch auf epigenetische Signaturen auswirken kann. Die funktionellen Konsequenzen dieser Veränderungen, darunter eine veränderte Expression der betroffenen Gene, wurden zudem als Modulatoren der psychobiologischen Stressantwort beschrieben. Eine Reihe erster Pionierstudien zeigt nun, dass stressassoziierte epigenetische Prozesse durch psychologische Interventionen, darunter Stressprävention und Stressbewältigung, verhindert oder sogar umgekehrt werden können. In der vorliegenden Übersichtsarbeit werden (1) relevante epigenetische Mecha- nismen beschrieben, (2) eine Übersicht zum Zusammenhang von Epigenetik und Stress dargestellt und (3) erläutert, wie epigenetische Prozesse durch psychologische Interventionen beeinflusst werden könnten.

(c) 2020 S. Karger AG, Basel

\section{Evaluation of Stress Management and Stress Prevention Using Epigenetic Markers}

\section{Keywords}

Epigenetics · Methylation · Stress management · Stress prevention

\section{Abstract \\ Stress is associated with mental health problems and physical diseases. Results from basic research indicate that stress-induced dysregulations of bodily stress response systems might mediate these processes. In consequence, many programs to prevent or manage stress have been developed and evaluated using endpoints of these bodily stress systems. Over the last few years, emerging evidence from human and animal studies has indicated that stress also leads to changes in epigenetic signatures. The func-}


tional consequences of these changes, among them altered gene expression, have been shown to modulate the psychobiological stress response. Some recent studies now show that psychosocial interventions, such as stress prevention and stress management, can alter epigenetic processes. In this review, we will present an outline on (1) relevant epigenetic mechanisms, (2) the current literature on the association of stress and epigenetics, and (3) mechanisms through which psychological interventions might alter stress-related epigenetic markers.

○ 2020 S. Karger AG, Basel

\section{Stress}

Stress ist eines der größten Gesundheitsprobleme in westlichen Gesellschaften. Untersuchungen der letzten Jahre zeigen, dass 23\% der in Deutschland lebenden Bevölkerung angaben, "häufig", und immerhin 38\%, "manchmal" Stress zu erleben [Techniker Krankenkasse, 2016]. Ergebnisse einer Querschnittsstudie aus dem Jahr 2013 mit 5'793 Befragten [Hapke et al., 2013] ergaben, dass 11\% aller Personen in Deutschland chronisch Stress erleben. In der Gesamtstichprobe zeigte sich zudem, dass ein geringes Alter in Kombination mit einem geringen sozioökonomischen Status zu einer erhöhten Prävalenz (17,7\%) von chronischem Stress führt. Bei geringer sozialer Unterstützung steigt die Prävalenz altersunabhängig sogar auf bis zu 26,2\%. Das Bundesministerium für Arbeit und Soziales veröffentlichte im Jahr 2018 in Zusammenarbeit mit der Bundesanstalt für Arbeitsschutz und Arbeitsmedizin einen Bericht, in dem stressassoziierte Störungen als häufigste Ursache für Arbeitsunfähigkeit im Jahr 2017 klassifiziert wurden [Bundesministerium für Arbeit und Soziales und Bundesanstalt für Arbeitsschutz und Arbeitsmedizin, 2018]. Aktuelle Schätzungen beziffern die durch Stress entstehenden Kosten für Gesundheitssysteme, Privatpersonen und Unternehmen im deutschsprachigen Raum (Schweiz und Deutschland) auf zwischen 4,64 und 29,24 Milliarden Euro [Elfering et al., 2018]. Die Weltgesundheitsorganisation (WHO) stellte bereits im Jahr 2005 fest, dass der Wandel, dem Menschen in den Industrienationen unterworfen sind, zu Stress führt, dass dieser Stress krank machen kann und dass dieser Befund aus gesundheitspolitischer Perspektive die Entwicklung von Präventions- und Behandlungsmaßnahmen nach sich ziehen muss [World Health Organization, 2006].

\section{Die psychobiologische Stressantwort}

Zentral für das Verständnis von Stress ist, dass die Stressantwort nicht rein psychisch, sondern vor allem auch über körperliche Mechanismen vermittelt wird.
Hans Selye und Walter B. Cannon haben bis heute wegweisende Modelle aufgestellt, die beschreiben, wie über Anpassung des körperlichen Aktivierungsniveaus ein Aufrechterhalten der körperlichen und psychischen Unversehrtheit im Angesicht eines bedrohlichen Stressors geschehen kann. Selye [2013] beschrieb die körperliche Stressantwort mit dem "Allgemeinen Anpassungssyndrom" in drei Phasen: (1) In der Alarmreaktion befindet sich der Körper in einer Art Schockzustand, in deren Folge die körperliche Aktivierung zunächst reduziert wird. In der Anti-Schockreaktion wird die körperliche Aktivierung dann stark erhöht. Im Rahmen des Anti-SchockStadiums lässt sich auch die von Cannon beschriebene "Fight-or-Flight"-Reaktion einordnen [Cannon, 1915]. Über eine Aktivierung des sympathischen Nervensystems werden vor allem über adrenerge Innervation für die "Fight-or-Flight"-Reaktion wichtige Zielsysteme aktiviert (u.a. Atmung, Durchblutung, Muskelspannung). Die weiteren Phasen des Modells erklären vor allem (2) die Anpassung an länger andauernde Stressoren (Phase der Resistenz) sowie (3) die Überlastung des Individuums bei zu langer Exposition (Phase der Erschöpfung).

In frühen Stress-Theorien waren Stressoren häufig physikalischer Natur, etwa extreme Kälte oder plötzliche Schmerzreize, die jeweils unmittelbar eine körperliche Stressreaktion auslösen. Die große Bedeutung psychischer Faktoren für die Aktivierung körperlicher Stresssysteme wurde erst in späteren Arbeiten näher erläutert und schließlich anerkannt. Dies führte beispielsweise zur Prägung des Begriffs "psychoendocrine", der für die Aktivierbarkeit der hormonellen Stressantwort durch psychologische Stimuli steht [z.B. Mason, 1971]. Seither sind einige körperliche Systeme bekannt geworden, die durch psychologischen Stress aktiviert werden. Am häufigsten untersucht und beschrieben sind zwei charakteristische körperliche Stressachsen [Gunnar und Quevedo, 2007]: Die erste dieser Achsen sorgt über die Aktivierung des Sympathikus für die Bereitstellung von Ressourcen (z.B. erhöhtes Herzzeitvolumen) während der "Fight-or-Flight"-Reaktion (sympatho-adrenomedulläres System). Die Aktivität der zweiten Stressachse, der Hypothalamus-Hypophysen-Nebennierenrinden-Achse (HHNA), wird erst mehrere Minuten nach Auftreten des Stressors messbar. Ihr Endprodukt, Cortisol, trifft in den Zielzellen auf Rezeptoren und stößt eine ganze Kaskade intrazellulärer Prozesse an, die letztendlich die Bereitstellung von Ressourcen (z.B. Bereitstellung von Glukose) und das Aufrechterhalten des psychischen und körperlichen Funktionsniveaus zum Ziel haben [Sapolsky et al., 2000]. Die Effekte von Stress im Körper sind jedoch noch weiter gefächert und berühren eine Vielzahl von weiteren Körpersystemen. So führt akuter Stress beispielsweise zu einer Hochregulation der unspezifischen Immunität, während chronischer Stress mit einer Reduktion der humoralen und zellulären Immunantwort 
assoziiert ist [Segerstrom und Miller, 2004]. Chronischer Stress beeinflusst zudem die strukturelle Plastizität in einigen Gehirnregionen (z.B. eine Atrophie der Dendriten und eine reduzierte Anzahl von Synapsen), die für die Regulation der Stressantwort von großer Relevanz sind [Hunter et al., 2015].

Die detaillierte Analyse der körperlichen Stressantwort zeigte, dass die Stressantwort über zentralnervöse emotionale und kognitive Mechanismen (neurobiologisch z.B. über die Aktivierung der Amygdala) reguliert wird. Zusammengefasst werden diese Erkenntnisse im "Allostase-Stress-Modell" [vgl. McEwen und Wingfield, 2003], das die kognitive Evaluation der Bedrohlichkeit von Situationen als entscheidend für die Entstehung von psychobiologischem Stress beschreibt. Das Modell integriert zudem (1) die Einflüsse interindividueller Dispositionen (etwa genetische Faktoren oder frühkindliche Erfahrungen) auf die Situationseinschätzung und die körperliche Stressantwort sowie (2) die wichtige Funktion von Verhalten als Moderator zwischen Situationseinschätzung und körperlicher Stressantwort. "Allostase" beschreibt den Zustand der "Stabilität durch Veränderung", also diejenigen psychobiologischen Prozesse, die dazu führen, dass sowohl kurz- als auch langfristig eine Anpassung an Stressoren stattfinden kann. Besonders wichtig für die Klinische Psychologie ist hier die sogenannte "allostatische Belastung". Sie beschreibt das Entstehen von Verschleiß- und Abnutzungserscheinungen ("wear and tear"), wenn eine dauerhafte und damit ressourcenintensive Anpassung an Stressoren notwendig wird. Die Folge kann beispielsweise eine Dysregulation der hormonellen Stressantwort oder eine gestörte Regulation des Immunsystems sein. Diese veränderte Allostase, also die Langzeitadaptation an einen oder mehrere Stressoren, wird über eine Reihe biologischer Mechanismen moderiert. Diese Mechanismen werden einerseits durch psychischen Stress aktiviert und sind andererseits Endpunkte der beschriebenen körperlichen Stresssysteme [Juster et al., 2010]. Solche Endpunkte können beispielsweise peripher-physiologisch (z.B. über die Ableitung eines Elektrokardiogramms) oder durch biochemische Assays (z.B. Cortisol im Speichel oder Immunparameter im Blut) bestimmt werden; entsprechend können diese Endpunkte in Studien zur Evaluation von psychologischen Interventionen herangezogen werden [Juster et al., 2010].

\section{Genetische und epigenetische Faktoren der Stressverarbeitung}

In den letzten Jahrzehnten wurden genetische Variationen (sogenannte Polymorphismen) von Kandidatengenen, die in die Regulation der körperlichen Stresssys- teme involviert sind, in Zusammenhang mit Stress und Psychopathologie intensiv untersucht. Erste Studien berichteten solche Zusammenhänge beispielsweise für das Serotonintransporter-Gen (SLC6A4) [siehe beispielsweise Karg et al., 2011], das Gen, das für das FK506-bindende Protein 51 kodiert (FKBP5) [siehe beispielsweise Zannas et al., 2016] sowie das Glucocorticoid-Rezeptor-Gen (NR3C1) [siehe beispielsweise Lian et al., 2014]. Die zitierten Arbeiten beschreiben stets sogenannte "Gen-Umwelt-Interaktionen". Ziel dieser Untersuchungen ist es zu bestimmen, ob genetische Dispositionen einen Einfluss darauf haben, wie vulnerabel ein Individuum ist, eine psychische Störung zu entwickeln, wenn es mit stressinduzierenden Umwelteinflüssen konfrontiert ist. Trotz der ursprünglich vielversprechenden Ergebnisse widerlegt nun eine aktuelle und große Übersichtsarbeit die Annahme, dass genetische Variationen in Kandidatengenen Einfluss auf die Vulnerabilität zur Entstehung depressiver Störungen haben könnten [Border er al., 2019]. In der gleichen Arbeit wurden zudem keine Nachweise für direkte Assoziationen von Kandidatengenen mit depressiven Störungen berichtet [Border et al., 2019]. Weitere genomweite Untersuchungen mit großen Fallzahlen bestätigen diese fehlenden Haupteffekte von Kandidatengenpolymorphismen [Wray et al., 2018; Howard et al., 2019] und berichten lediglich kleine Effektstärken für die direkte Assoziation von 102 unabhängigen genetischen Variationen [Howard et al., 2019] und 44 Genloci [Wray et al., 2018] mit depressiven Störungen. Auch für weitere psychische Störungen, wie beispielsweise Schizophrenie, sind Ergebnisse $\mathrm{zu}$ Kandidatengen-Umwelt-Interaktionen häufig nicht replizierbar [Assary et al., 2018]. Dies könnte darin bergründet liegen, dass die Analyse von Kandidatengenen den polygenen Charakter psychischer Störungen, also das Zusammenspiel mehrerer Gene, vernachlässigt [Assary et al., 2018]. Die Komplexität der Genese stressassoziierter psychischer Störung durch genetische Variationen zu erklären, ist bisher folglich nur eingeschränkt gelungen.

Unstrittig ist jedoch die Wichtigkeit von Umweltfaktoren bei der Entstehung psychischer Störungen [Border et al., 2019]. Erst seit einigen Jahren ist bekannt, dass Stressoren Umweltfaktoren sein können, über die eine Veränderung der Genaktivität bei Genen in wichtigen Zielsystemen vermittelt wird [siehe zum Beispiel Zannas und West, 2014]. Die Prozesse, über die solch ein Einfluss vermittelt wird, werden dem Fachgebiet der "Epigenetik" zugeschrieben. Dieses Fachgebiet befasst sich mit molekularbiologischen Prozessen, welche - unabhängig von der DNA-Sequenz - einen Einfluss auf die Aktivierbarkeit eines Gens nehmen können [Wu und Morris, 2001]. Die aus diesen Prozessen resultierenden epigenetischen Zustände werden im Prozess der Zellteilung vererbt, sind jedoch potenziell reversibel [Schuebel et al., 2016]. Die 
wichtigsten und im Zusammenhang mit Stress am häufigsten untersuchten epigenetischen Mechanismen sollen im Folgenden kurz erläutert werden.

\section{Struktur der DNA}

Der DNA-Strang liegt im Zellkern nicht unstrukturiert vor. Kurze Abschnitte des Strangs sind jeweils um Komplexe aus acht Histon-Proteinen gewickelt [Portela und Esteller, 2010]. Die so entstehenden Komplexe werden als Nukleosome bezeichnet [Portela und Esteller, 2010]. Für die Aktivierbarkeit von Genen sind unter anderem bestimmte epigenetische Modifikationen entscheidend, die hauptsächlich an den unstrukturierten Enden dieser Proteine (sogenannte "Histonschwänze") stattfinden. Eine Kombination und Interaktion aus mehreren dieser epigenetischen Veränderungen führt dazu, dass die DNA dichter oder weniger dicht um die Histone gewickelt ist. Solche Mechanismen sind beispielsweise Phosphorylierung, Methylierung oder Acetylierung sowie deren jeweilige Gegenteile (etwa Deacetylierung durch Histon-Deacetylasen) [Schroeder et al., 2012]. Eine stärkere Verdichtung des Komplexes führt zu "Heterochromatin", während eine Entfaltung zu "Euchromatin" führt. Im entfalteten und weniger dicht verpackten $\mathrm{Zu}$ stand ist ein besserer Zugang von bestimmten Proteinen (sogenannten "Transkriptionsfaktoren") möglich, die wichtig für die Initiation der Genexpression (sogenannte "Transkription") sind. Dies führt zu einer besseren Aktivierbarkeit des betroffenen Gens [Schroeder et al., 2012]. Neben diesen sogenannten "Histon-Modifikationen" ist von Bedeutung, wie die Verbindungen aus DNA-Strang und Proteinen, also die Nukleosome, im Zellkern angeordnet sind. Dies gilt besonders für den Bereich des Transkriptionsstarts, da dort ein Komplex aus Enzymen (RNA-Polymerase) bindet, der das Erbgut abliest und somit die Synthese von neuen Proteinen initiiert. Epigenetische Prozesse, die Einfluss auf die Anordnung der $\mathrm{Nu}$ kleosome nehmen, sind vielfältig. Besonders wichtig sind jedoch ATP-abhängige Enzymkomplexe ("chromatin remodeling complexes"), welche Nukleosom repositionieren, destabilisieren oder abstoßen können [Ho und Crabtree, 2010].

\section{DNA-Methylierung}

Weitaus häufiger als epigenetische Prozesse, welche die Struktur der DNA beeinflussen, ist besonders in der psychiatrischen Forschung die DNA-Methylierung untersucht, weswegen sich die vorliegende Übersichtsarbeit vorwiegend mit diesem Prozess befassen wird. DNA-Methylierung ist ein Prozess, bei dem bestimmte Enzyme (sogenannte Methylgruppen) an Bausteine der DNA (z.B. das Cytosin von Cytosin-Phosphat-Guanin-Dinkuleotiden [CpG-Dinukleotide] aber auch von CpA-, CpC- oder CpT-Dinukleotiden) angehängt werden [Portela und Es- teller, 2010]. Das methylierte Cytosin wird zu 5-Methylcytosin und hat, vor allem in Bereichen, in denen CpGDinukleotide gehäuft vorkommen ("CpG-Inseln"), eine reduzierte Aktivierbarkeit von Genen zur Folge, das sogenannte "Silencing" [Jones, 2012]. CpG-Methylierung führt vor allem im Bereich des Transkriptionsstarts zu einer reduzierten Aktivierbarkeit von Genen, da dort die Bindung von Transkriptionsfaktoren sowie die Initiation der Transkription eingeschränkt werden [Jones, 2012]. Es konnte zudem gezeigt werden, dass auch Methylierung im Genkörper, also nicht im Bereich des Transkriptionsstarts, Relevanz für die Transkription haben kann [Varley et al., 2013]. Ferner bewirkt Methylierung an CpG-Dinukleotiden auch die Rekrutierung bestimmter Proteinkomplexe, die zu strukturellen Veränderungen der DNA führen können (Histon-Modifikationen und Veränderungen der Chromatinstruktur) [Bogdanović und Veenstra, 2009]. Entsprechend der oben beschriebenen Funktionen dieser epigenetischen Prozesse führt dies erneut zu einer veränderten Aktivierbarkeit der Gene.

\section{Epigenetik und Stress}

Vor etwa 15 Jahren wurde in einer ersten Studie [Weaver et al., 2004] der Nachweis dafür erbracht, dass auch körperliche Systeme und Regulationsmechanismen, die an der psychobiologischen Stressantwort beteiligt sind, durch epigenetische Prozesse beeinflusst werden können. Die Autoren konnten bei Ratten nachweisen, dass ein reduziertes mütterliches Versorgungsverhalten beim Nachwuchs die Methylierung von $\mathrm{Nr} 3 \mathrm{cl}$ veränderte, eine veränderte Transkriptionsfaktor-Bindung (NGFI-A) bedingte, Einfluss auf die Histon-Acetylierung nahm und, vermutlich in Folge dieser Prozesse, zu einer veränderten Nr3c1-Expression führte. In Übereinstimmung damit konnten die Autoren nachweisen, dass das mütterliche Versorgungsverhalten beim Nachwuchs die HHNA-Reaktivität auf akuten Stress beeinflusste. Diese Studie war wegweisend für das Entstehen eines neuen Forschungsfeldes, das Verhaltensmuster in Assoziation zu Veränderungen der epigenetischen Signatur relevanter Kandidatengene setzte, das Feld der "behavioral epigenetics" [Lester et al., 2011]. Zahlreiche Studien haben seither auch bei Menschen - zeigen können, dass eine veränderte Exposition zu Umwelteinflüssen einen Einfluss auf epigenetische Prozesse haben kann. So zeigen mehrere Reviews Einflüsse von Stress auf epigenetische Prozesse in Genen, die für die psychische Gesundheit von Relevanz sind. Darunter fallen besonders pränataler [Provenzi et al., 2018], frühkindlicher [Jiang et al., 2019] und in frühen Lebensphasen erlebter Stress [Vaiserman, 2015]. Eine Vielzahl der Publikationen im Bereich der behavioralen 
Epigenetik fokussiert auf pränatal oder frühkindliche Einflussfaktoren, weil in diesen Entwicklungsphasen eine besonders hohe Vulnerabilität und Plastizität besteht [Pérez et al., 2019]. Die Ergebnisse zu diesen Einflüssen können aufgrund der Menge an Befunden als robust repliziert betrachtet werden.

Es ist aber bereits seit einigen Jahren bekannt, dass epigenetische Prozesse auch im Erwachsenenalter noch veränderbar sind [Sweatt, 2009], da die zur Methylierung notwendigen Enzyme (Methyltransferasen) auch im erwachsenen Gehirn weiterhin aktiv sind [Feng et al., 2010]; auch in Studien an Menschen konnte dies bereits nachgewiesen werden [z.B. Siegmund et al., 2007]. In Bezug auf Stress zeigen mehrere Befunde, dass vor allem länger andauernde Phasen der Stressexposition (zum Beispiel arbeitsbezogener, traumatischer, sozioökonomisch bedingter oder chronischer Stress) im Erwachsenenalter die Methylierung verschiedenster Kandidatengene verändern können [Unternaehrer und Meinlschmidt, 2016; Bakusic et al., 2017; Park et al., 2019]. Einige frühere Studien legen zudem nahe, dass im Erwachsenenalter aktive Methyltransferasen konkret an der Steuerung neuronaler und behavioraler Adaptionen beteiligt sind, darunter an Gedächtnisprozessen [Heyward und Sweatt, 2015], an der mit zunehmendem Alter abnehmenden kognitiven Leistungsfähigkeit [Oliveira et al., 2012] sowie an depressivem Verhalten [LaPlant et al., 2010]. In zwei Studien an Ratten konnten ferner erste Nachweise dafür erbracht werden, dass sogar Akutstress einen konkreten Einfluss auf die Methylierung bestimmter Kandidatengene [Miller und Sweatt, 2007] sowie auf die globale DNA Methylierung [Rodrigues et al., 2015] ausüben könnte. Eine neuere Studie an erwachsenen Ratten zeigte zudem eindrücklich, dass ein Enzym zur Methylierung nach einer Akutstressexposition vermehrt an Nr3c1 gebunden war, was eine aktive Methylierung zur Folge hatte und zu einer geringeren Aktivierung des Gens führte [Mifsud et al., 2017]. Darüber hinaus konnte bisher zwei Mal gezeigt werden, dass chronischer Stress zu einer veränderten Aktivierung von Enzymen führt, welche die Methylierung von unter anderem stressrelevanten Genen steuern [Elliott et al., 2010; LaPlant et al., 2010]. Interessanterweise legen nun auch die Ergebnisse einer ersten Studie an Menschen nahe, dass akuter psychosozialer Stress mit einem Anstieg der Methylierung des Oxytocin-RezeptorGens (OXTR) einhergehen könnte [Unternaehrer et al., 2012]. Da die Methylierung von OXTR selbst funktionell mit der HHNA-Reaktivität auf akuten psychosozialen Stress assoziiert ist [Ziegler et al., 2015], kann auch in diesem Fall vermutet werden, dass epigenetische Prozesse eine schnelle Anpassung an den Akutstress vermittelten. In der Zusammenschau deuten diese Befunde darauf hin, dass Methylierung in ausgereiften Zellen weiterhin möglich und vermutlich ein entscheidender Faktor für die
Plastizität und Adaptationsfähigkeit des erwachsenen Gehirns ist [Bayraktar und Kreutz, 2018]. In den vorstehend zitierten Arbeiten wird dabei eine Reihe von Kandidatengenen besonders häufig genannt. Darunter fallen neben NR3C1 [siehe Turecki und Meaney, 2016 für eine Übersicht], SLC6A4 [siehe Palma-Gudiel und Fañanás, 2017 für eine Übersicht] sowie FKBP5 [siehe Zannas et al., 2016 für eine Übersicht]. Den zitierten Übersichtsarbeiten ist zu entnehmen, dass epigenetische Prozesse in allen drei Genen an der Regulation der HHNA beteiligt sind. Sie kovariieren zudem mit funktionellen Prozessen und/oder strukturellen Veränderungen in Gehirnregionen, die zentral an der Steuerung der körperlichen Stressantwort beteiligt sind [Nikolova und Hariri, 2015].

\section{Reversibilität stressassoziierter epigenetischer Signaturen}

Für die Annahme, dass epigenetische Mechanismen nicht nur pränatal oder frühkindlich geschehen, sondern auch im erwachsenen Gehirn weiter vonstattengehen, sprechen ferner Ergebnisse der Studie von Weaver und Kollegen [2004] (siehe Kapitel 4). Die Autoren konnten nachweisen, dass diejenigen epigenetischen Prozesse in $N r 3 c 1$, die durch fehlendes mütterliches Versorgungsverhalten induziert waren, durch die Gabe eines Histon-Deacetylase-Inhibitors nichtig gemacht werden konnten. Diese "proof of concept"-Demonstration der Reversibilität von behavioral geprägten epigenetischen stressassoziierten Signaturen ist zentral für die Annahme eines hochdynamisches Epigenoms, das nicht nur auf Stress, sondern auch auf die Bewältigung von Stress oder das Fördern von Resilienz reagieren könnte [Zannas und Binder, 2014].

Für die Evaluation psychotherapeutischer Interventionen haben diese Befunde unmittelbare Relevanz: So gibt es erste longitudinale Studien, in welchen epigenetische Signaturen von Kandidatengenen bei Menschen mit psychischen Störungen vor und nach psychotherapeutischen Interventionen untersucht wurden. Diese Studien berichten tatsächlich eine Co-Variation von Symptomverbesserung und Veränderungen in der epigenetischen Signatur von $B D N F$ (ein Gen, das für einen Wachstumsfaktor kodiert) [Perroud et al., 2013], FKBP5 [Yehuda et al., 2013; Roberts et al., 2015, 2019] und SLC6A4 [Roberts et al., 2014]. Obwohl bisher nur wenige Studien zur Reversibilität von stressassoziierten epigenetischen Signaturen existieren, scheint die Messung ebendieser also vielversprechend, gerade um die Wirksamkeit von Stressprävention und Stressbewältigung zu evaluieren. Eine steigende Resilienz, im Sinne von gesundheitsförderlichen Interpretations- und Verhaltensmustern bei Belastung, könnte dabei über eine Neuausrichtung maladaptiver 
epigenetischer Signaturen vermittelt werden [McEwen, 2016]. Langfristig könnte sich folglich die Möglichkeit bieten, Interventionen zur Förderung von Resilienz oder zur Bewältigung von Stress gezielt zu diesem Zwecke einzusetzen und die Veränderung in der epigenetischen Signatur als Maß für deren Wirksamkeit zu interpretieren [Szyf et al., 2016].

\section{Stressprävention und Stressbewältigung}

Es gibt eine ganze Reihe von Interventionen, die zur Prävention oder Bewältigung von Stress entwickelt wurden. Für viele dieser Interventionen, unter anderem Entspannungsverfahren [Esch et al., 2003], Psychoedukation [Van Daele et al., 2012] oder kognitiv-behavioralen Verfahren, ist eine gute Wirksamkeit nachgewiesen [Varvogli und Darviri, 2011]. Darüber hinaus sind auch übergeordnete Einflussfaktoren bekannt, die Stress reduzieren oder verhindern können, wie zum Beispiel soziale Unterstützung [Ditzen und Heinrichs, 2014], Sport und ausreichender und regelmäßiger Schlaf. Hervorzuheben sind achtsamkeitsbasierte Verfahren, die nicht nur mit Abstand am besten untersucht sind, sondern für die auch die belastbarsten Evidenzen für gesundheitsförderliche Effekte vorliegen [Gu et al., 2015]; darunter besonders Effekte auf die Aktivierung verschiedener an der Stressreaktion beteiligter Gehirnareale [Tang et al., 2015].

Erst in den letzten Jahren sind erste Arbeiten veröffentlich worden, die nahelegen, dass die gesundheitsförderlichen Effekte von Achtsamkeitspraxis zum Teil epigenetisch vermittelt sein könnten. In einer erst kürzlich erschienenen Studie wurde die epigenomweite Methylierung bei insgesamt 34 Personen untersucht, von denen 17 mindestens 10 Jahre Meditationserfahrung vorzuweisen hatten [García-Campayo et al., 2018]. Die Ergebnisse zeigten 64 differentiell methylierte Regionen auf insgesamt 43 Genen, von denen etwa die Hälfte mit körperlichen Erkrankungen und psychischen Störungen in Verbindung steht.

In einer früheren Studie wurden die Effekte einer eintägigen achtsamkeitsbasierten Intervention in einer Gruppe von 19 meditationserfahrenen Teilnehmern (>3 Jahre Erfahrung) untersucht. Als Kontrollgruppe fungierten 21 meditationsunerfahrene Teilnehmer, die im Interventionszeitraum an Freizeitaktivitäten teilnahmen. Alle Parameter wurden in beiden Gruppen jeweils einmal vor und einmal nach der Intervention (bzw. der Freizeitaktivitäten) erhoben. Im Vergleich zur Kontrollgruppe zeigten sich in der Interventionsgruppe über die Zeit eine reduzierte Expression von Histon-Deacetylase-Genen (HDAC2, HDAC3, HDAC9) und proinflammatorischen Genen (RIPK2 und COX-2) sowie globale Modifikationen an Histonen (H4ac und H3K4me3) [Kaliman et al.,
2014]. Die Autoren berichten zudem, dass die Expression von RIPK2 und HDAC2 in der Gesamtstichprobe mit einer reduzierten HNNA-Reaktivität auf akuten psychosozialen Stress assoziiert war. In einer epigenomweiten Analyse der gleichen Stichprobe stellten die Autoren zudem nur in der Interventionsgruppe an 61 CpG-Dinukleotiden signifikante Veränderungen der Methylierung über die Zeit der Intervention fest [Chaix et al., 2019]. Die 61 Dinukleotide wurden als überzufällig häufig in CpGInseln lokalisiert beschrieben und Genen zugeordnet, die Funktionen in der Steuerung des Immunsystems und des Alterns haben. In einer querschnittlichen Analyse der gleichen Stichprobe konnten die Autoren ferner nachweisen, dass sich die altersbedingte Beschleunigung epigenetischer Alterungsprozesse ("epigenetic clock") ab dem 52. Lebensjahr in 17 der meditationserfahrenen Probanden nicht in gleichem Maße zeigte wie in der Kontrollgruppe nicht-meditationserfahrener Probanden [Chaix et al., 2017]. Das Konzept der in der Studie beschriebenen "epigenetischen Uhr" beschreibt, dass - anhand der Methylierung von 353 CpG-Dinukleotiden ein epigenetisches Alter bestimmt werden kann, das eine Vielzahl von Gesundheitsparametern vorhersagt [Horvath, 2013].

Eine neuere Studie an 22 traumatisierten Kriegsveteranen fokussierte auf ein standardisiertes "MindfulnessBased Stress-Reduction"-Programm (MBSR) [Bishop et al., 2018]. Die Autoren berichten, dass bei 11 Veteranen begleitend zur Reduktion der Trauma-Symptome durch die MBSR-Intervention eine Reduktion der FKBP5-Methylierung stattfand. Die Autoren berichten allerdings keinen solchen Effekt für die Methylierung von SLC6A4, das ebenfalls in der Studie untersucht wurde. Obwohl es weiterer und größerer Studien bedarf, um eine endgültige Aussage zu den einzelnen epigenetischen Veränderungen zu treffen, so sind die bisherigen Hinweise dafür, dass achtsamkeitsbasierte Verfahren einen messbaren Effekt auf epigenetische Prozesse haben, hochinteressant [siehe Kaliman, 2019].

In einer eigenen Studie an einer Stichprobe von Medizinstudierenden mit Fokus auf epigenetische Prozesse in SLC6A4 konnten wir kürzlich zeigen, dass sich die Methylierung durch eine achtsamkeitsbasierte Intervention über drei Monate hinweg beeinflussen lässt [siehe Stoffel et al., 2019a für Details]. Die Methylierung wurde in dieser Studie auf 56 CpG-Dinukleotiden untersucht, die in einem Bereich lokalisiert liegen, in dem epigenetische Prozesse bedeutsam für die Aktivierbarkeit des Gens sind (sogenannte "Promoter-assoziierte" Region). Die mittlere Methylierung nahm über die drei Monate in der Interventionsgruppe ab und blieb in einer Kontrollgruppe nahezu unverändert. Da eine geringere Methylierung in genau dem Bereich, den wir in der Studie untersuchten, nachweisbar mit geringerem Stress assoziiert ist 
[Palma-Gudiel und Fañanás, 2017], lässt sich auf einen stressprotektiven Effekt dieser Veränderungen schließen. Diese Vermutung wird vor allem dadurch gestützt, dass eine stärkere Abnahme der Methylierung mit einer stärkeren Reduktion des subjektiven Stresserlebens und der Selbsteinschätzung, grundsätzlicher besser mit Stress umgehen zu können, assoziiert war. Zudem war die Reduktion der Methylierung bei den Probanden stärker, die außerhalb der eigentlichen Interventionssitzungen im Alltag Stressbewältigungsübungen durchgeführt hatten. Zuletzt zeigten die Ergebnisse, dass eine Reduktion der Methylierung tatsächlich eine funktionelle Relevanz für den Serotoninstoffwechsel haben könnte, da eine Abnahme der Methylierung mit einer Zunahme der Expression von SLC6A4 assoziiert war, was für eine stärkere Aktivierung des Gens spricht. Entsprechend dieser Ergebnisse nahm die SLC6A4 Expression über die drei Monate in der Interventionsgruppe signifikant stärker $\mathrm{zu}$ als in der Kontrollgruppe. Interessant ist ebenfalls, dass häufig untersuchte strukturelle genetische Variationen in SLC6A4 keinen Einfluss auf die beschriebenen Ergebnisse zeigten.

In einer weiteren eigenen Studie haben wir eine Stressbewältigungsintervention untersucht, die unter anderem auf Techniken der lösungsorientierten Kurzzeittherapie im Umgang mit Stressoren zurückgreift [De Shazer und Dolan, 2016] und explizit das Nutzen sozialer Kontakte als Ressourcen fördert. Die Intervention mit dem Titel "Der Grüne Bereich" [Ditzen und Ehlert, 2014] wurde in mehreren Gruppen von Arbeitnehmern an jeweils zwei Tagen im Abstand einer Woche durchgeführt. Jeweils vor und nach der Intervention wurden pro Proband einmal die Methylierung von FKBP5 und NR3C1 sowie die Ausschüttung von Cortisol im Alltag erhoben. Erste Ergebnisse deuten darauf hin, dass die Methylierung von FKBP5 innerhalb der Woche zwischen beiden Messungen in der Interventionsgruppe signifikant zunahm, während sie sich in der Kontrollgruppe nicht veränderte [Stoffel et al., 2017]. Darüber hinaus war die Methylierung an zwei der fünf untersuchten CpG-Dinukleotide von FKBP5 signifikant mit der zirkadianen Rhythmik von Cortisol assoziiert [Stoffel et al., 2019b]. Zusammengenommen zeigt sich, dass epigenetische Prozesse - gerade in wichtigen Kandidatengenen wie FKBP5 oder SLC6A4 - nicht nur hochreaktiv auf Stress reagieren, sondern vermutlich auch durch die Prävention oder Bewältigung von Stress beeinflusst werden können. Aufgrund der wichtigen Funktionen, welche diese Gene im Kontext der Stressantwort spielen, könnten sie sich deshalb sehr gut zur Evaluation von Interventionen eignen, die die Stresssysteme adressieren. Künftige Studien müssen zeigen, ob sich die in dieser Übersichtsarbeit vorgestellten ersten Nachweise robust replizieren lassen.

\section{Grenzen der Evaluation von Stressbewältigung und Stressprävention mittels epigenetischer Marker}

Grundlegend ist das Forschungsfeld, das sich mit der Evaluation psychologischer Interventionen mittels epigenetischer Marker befasst, noch sehr heterogen. Dies betrifft beispielsweise die untersuchten Gene, die angewendeten biochemischen Methoden [siehe Kurdyukov und Bullock, 2016 für eine Übersicht], die Datenverarbeitung, die untersuchten Populationen und stressassoziierten Störungen sowie die untersuchten Interventionen. Diese Heterogenität erschwert die Generalisierbarkeit der bisherigen Befunde.

In den Kapiteln 3 bis 6 wurden beispielhaft die drei Gene SLC6A4, FKBP5 und NR3C1 herausgestellt, die sich aufgrund der Tatsache, dass sie bereits vielfach in Zusammenhang mit Stress untersucht wurden, als Kandidatengene der Stressforschung anbieten [siehe Bakusic et al., 2017; Park et al., 2019 für eine Übersicht und weitere Kandidatengene]. Nichtsdestotrotz weist besonders der Kandidatengen-Ansatz, der bisher in den meisten Studien zum Einsatz kommt, methodische Schwierigkeiten auf: So konnte beispielsweise gezeigt werden, dass ein hohes Maß an Kovariation der Methylierung zwischen Untereinheiten von CpG-Dinukleotiden über das gesamte Genom hinweg existiert. Für diese Kovariation kann nur in epigenomweiten Analysen ausreichend kontrolliert werden, da in Kandidatengen-Studien nur jeweils wenige CpG-Dinukleotide analysiert werden (im Vergleich zu bis ca. 850'000 in epigenomweiten Analysen) [Shabalin et al., 2015]. Die alternative Nutzung von epigenomweiten Analysen ist zwar auch in einem longitudinalen Interventionsdesign denkbar, würde jedoch spezifische Herausforderungen mit sich bringen [Michels et al., 2013], darunter vor allem die Notwendigkeit sehr großer Stichproben, die aktuell noch hohen Kosten und die möglicherweise geringe Abdeckung von CpGDinukleotiden auf relevanten Genen durch das genutzte Array.

Eine weitere Limitation ist dadurch gegeben, dass das Gewebe des bei der Erforschung psychologischer Prozesse relevanten Zielorgans, des Gehirns, nur post mortem zugänglich ist. Folglich müssen meist in der Körperperipherie verfügbare Gewebe für epigenetische Analysen genutzt werden; hierfür kommen häufig peripheres Blut, Speichelproben oder Zellen aus der Mundschleimhaut zum Einsatz. Da Methylierung jedoch zellen- und gewebespezifisch sein kann, kann die Wahl des Gewebes zur Extraktion der genomischen DNA Einfluss auf die Ergebnisse der jeweiligen Studie haben [Zhang et al., 2013]. Um festzustellen, inwiefern die Methylierung im Gehirn mit der Methylierung von Gewebe in der Peripherie assoziiert ist, wurden datenbankbasierte Online-Tools entwickelt, mit denen ein direkter Vergleich möglich 
wird [z.B. Braun et al., 2019]. Um den Einfluss der Zellzusammensetzung auf Methylierungsdaten zu berücksichtigen, können beispielsweise statistische Verfahren genutzt werden [z.B. Jones et al., 2017].

\section{Schlussfolgerung und Ausblick}

In Übereinstimmung mit einer früheren Übersichtsarbeit, welche sich mit der Rolle epigenetischer Prozesse für den Bereich der Gesundheitsförderung im Allgemeinen befasste [McBride und Koehly, 2017], kann Folgendes festgestellt werden: (1) Epigenetische Faktoren werden durch Umwelteinflüsse aktiv und flexibel verändert. Die Messung dieser Veränderungen hat großes Potential, künftig als Biomarker zu fungieren. Gerade epigenetische Signaturen auf Kandidatengenen, bei denen eine Assoziation mit einem der Zielkonstrukte - Stress oder Resilienz - stabil nachgewiesen ist, bieten sich dafür an. Systematische Untersuchungen der Sensitivität und Spezifität epigenetischer Marker zur Vorhersage der Zielkonstrukte müssen in Zukunft zeigen, ob die Kriterien zur Verwendung epigenetischer Signaturen als Biomarker formell erfüllt werden können. (2) Als gezielte Umwelteinflüsse könnten Interventionen zur Förderung von Gesundheit epigenetische Muster ebenfalls modifizieren: Sofern ausreichend Evidenzen für die Kovariation einer bestimmten epigenetischen Signatur mit einem der Zielkonstrukte vorläge, könnten Veränderungen in ebendieser Signatur künftig als objektiver Indikator für den Erfolg der untersuchten Interventionen genutzt werden. Spezifisch in Bezug auf Stress zeigen die in der vorliegenden Übersichtsarbeit dargestellten Studien erste Hinweise dafür, dass stressabhängige Veränderungen epigenetischer Signaturen durch Stresspräventions- oder Stressbewältigungsintervention - darunter besonders achtsamkeitsbasierte Interventionen - tatsächlich abgepuffert werden können. Zum weiterführenden Verständnis des Zusammenhangs von Stress und epigenetischen Veränderungen wäre es in Zukunft jedoch hochinteressant, zusätzlich epigenomweite Assoziationsstudien durchzuführen [siehe Rakyan et al., 2011]. Kandidatengene erfüllen zwar häufig wichtige Funktionen in regulatorischen Systemen der psychobiologischen Stressantwort, decken jedoch bei Weitem nicht alle durch Stress veränderten Gesundheitsparameter ab. Zusätzlich wäre es dann möglich, epigenetische Profile - im Sinne einer personalisierten Versorgung - als Prädiktoren des Interventionserfolges zu untersuchen.

Langfristig könnte die beschriebene Entwicklung darin münden, dass auch die Erfolge von Psychotherapie über den Selbstbericht und die Verhaltensbeobachtung hinaus - auf epigenetischer Ebene objektivierbar werden. Die Ergebnisse der in dieser Übersichtsarbeit vorgestellten ersten Studien deuten schließlich darauf hin, dass psychologische Interventionen möglicherweise Einfluss auf die Regulation stressassoziierter epigenetischer Prozesse haben könnten. Künftige randomisiert-kontrollierte Interventionsstudien müssen zeigen, ob diese Annahme haltbar sein wird.

\section{Acknowledgments}

Die Arbeit wurde finanziell durch Mittel des Ministeriums für Wissenschaft, Forschung und Kunst Baden-Württemberg gefördert. Die fördernde Organisation hatte keinerlei Einfluss auf die Entstehung der Arbeit.

\section{Disclosure Statement}

Es bestehen keine Interessenkonflikte im Zusammenhang mit der Übersichtsarbeit. Die Übersichtarbeit basiert auf Teilen der Dissertation "Psychobiologische Evaluation eines Stressbewältigungstrainings im Alltag", verfasst von Martin Stoffel, die im September 2019 bei der Medizinischen Fakultät Heidelberg der Universität Heidelberg zur Erlangung des Doctor scientiarum humanarum (Dr. sc. hum.) eingereicht wurde.

\section{Literatur}

Assary E, Vincent JP, Keers R, Pluess M. Geneenvironment interaction and psychiatric disorders: review and future directions. Semin Cell Dev Biol. 2018 May;77:133-43.

Bakusic J, Schaufeli W, Claes S, Godderis L. Stress, burnout and depression: A systematic review on DNA methylation mechanisms. J Psychosom Res. 2017 Jan;92:34-44.

Bayraktar G, Kreutz MR. Neuronal DNA Methyltransferases: Epigenetic Mediators between Synaptic Activity and Gene Expression? Neuroscientist. 2018 Apr;24(2):17185.

Bishop JR, Lee AM, Mills LJ, Thuras PD, Eum S, Clancy D, et al. Methylation of FKBP5 and SLC6A4 in Relation to Treatment Response to Mindfulness Based Stress Reduction for
Posttraumatic Stress Disorder. Front Psychiatry. 2018 Sep;9:418.

Bogdanović O, Veenstra GJ. DNA methylation and methyl-CpG binding proteins: developmental requirements and function. Chromosoma. 2009 Oct;118(5):549-65.

Border R, Johnson EC, Evans LM, Smolen A, Berley N, Sullivan PF, et al. No Support for Historical Candidate Gene or Candidate Geneby-Interaction Hypotheses for Major Depression Across Multiple Large Samples. Am J Psychiatry. 2019 May;176(5):376-87.

Braun PR, Han S, Hing B, Nagahama Y, Gaul LN, Heinzman JT, et al. Genome-wide DNA methylation comparison between live human brain and peripheral tissues within individuals. Transl Psychiatry. 2019 Jan;9(1):47.
Bundesministerium für Arbeit und Soziales, Bundesanstalt für Arbeitsschutz und Arbeitsmedizin: Sicherheit und Gesundheit bei der Arbeit - Berichtsjahr 2017. Unfallverhütungsbericht Arbeit. Dortmund, Kettler, 2018. https://www.baua.de/DE/Angebote/Publikationen/Berichte/Suga-2017.pdf\%3F_blob\%3 DpublicationFile\%26v\%3D13

Cannon WB. Bodily changes in pain, hunger, fear and rage: An account of recent researches into the function of emotional excitement. New York: D. Appleton \& Company; 1915. https:// doi.org/10.1037/10013-000.

Chaix R, Alvarez-López MJ, Fagny M, Lemee L, Regnault B, Davidson RJ, et al. Epigenetic clock analysis in long-term meditators. Psychoneuroendocrinology. 2017 Nov;85:210-4. 
Chaix R, Fagny M, Cosin-Tomás M, AlvarezLópez M, Lemee L, Regnault B, et al. Differential DNA methylation in experienced meditators after an intensive day of mindfulnessbased practice: implications for immune-related pathways. Brain Behav Immun. 2019 Nov 13. pii: S0889-1591(19)308797.

De Shazer S, Dolan Y. Mehr als ein Wunder: Die Kunst der lösungsorientierten Kurzzeittherapie. Heidelberg: Carl-Auer Verlag GmbH; 2016.

Ditzen B, Ehlert U. Stress und stressabhängige körperliche Störungen. In: Vaitl D, Petermann F, editors. Entspannungsverfahren. Das Praxishandbuch. Weinheim: Beltz; 2014. pp. 175-89.

Ditzen B, Heinrichs M. Psychobiology of social support: the social dimension of stress buffering. Restor Neurol Neurosci. 2014;32(1):14962 .

Elfering A, Brunner B, Igic I, et al. Gesellschaftliche Bedeutung und Kosten von Stress. In: Fuchs R, Gerber M, editors. Handbuch Stressregulation und Sport. Berlin, Heidelberg: Springer Berlin Heidelberg; 2018. pp. 123-41.

Elliott E, Ezra-Nevo G, Regev L, Neufeld-Cohen A, Chen A. Resilience to social stress coincides with functional DNA methylation of the Crf gene in adult mice. Nat Neurosci. 2010 Nov;13(11):1351-3.

Esch T, Fricchione GL, Stefano GB. The therapeutic use of the relaxation response in stress-related diseases. Med Sci Monit. 2003 Feb; 9(2):RA23-34.

Feng J, Zhou Y, Campbell SL, Le T, Li E, Sweatt JD, et al. Dnmt1 and Dnmt3a maintain DNA methylation and regulate synaptic function in adult forebrain neurons. Nat Neurosci. 2010 Apr;13(4):423-30.

García-Campayo J, Puebla-Guedea M, Labarga A, Urdánoz A, Roldán M, Pulido L, et al. Epigenetic Response to Mindfulness in Peripheral Blood Leukocytes Involves Genes Linked to Common Human Diseases. Mindfulness (N Y). 2018;9(4):1146-59.

Gu J, Strauss C, Bond R, Cavanagh K. How do mindfulness-based cognitive therapy and mindfulness-based stress reduction improve mental health and wellbeing? A systematic review and meta-analysis of mediation studies. Clin Psychol Rev. 2015 Apr;37:1-12.

Gunnar M, Quevedo K. The neurobiology of stress and development. Annu Rev Psychol. 2007;58(1):145-73.

Hapke U, Maske UE, Scheidt-Nave C, Bode L, Schlack R, Busch MA. Chronischer Stress bei Erwachsenen in Deutschland: Ergebnisse der Studie zur Gesundheit Erwachsener in Deutschland (DEGS1). Bundesgesundheitsblatt Gesundheitsforschung Gesundheitsschutz. 2013 May;56(5-6):749-54.

Heyward FD, Sweatt JD. DNA Methylation in Memory Formation: emerging Insights. Neuroscientist. 2015 Oct;21(5):475-89.

Ho L, Crabtree GR. Chromatin remodelling during development. Nature. 2010 Jan;463(7280): 474-84.

Horvath S. DNA methylation age of human tissues and cell types. Genome Biol. 2013, 14(10):R115.
Howard DM, Adams MJ, Clarke TK, Hafferty JD, Gibson J, Shirali M, et al.; 23andMe Research Team; Major Depressive Disorder Working Group of the Psychiatric Genomics Consortium. Genome-wide meta-analysis of depression identifies 102 independent variants and highlights the importance of the prefrontal brain regions. Nat Neurosci. 2019 Mar;22(3): 343-52.

Hunter RG, Gagnidze K, McEwen BS, Pfaff DW. Stress and the dynamic genome: Steroids, epigenetics, and the transposome. Proc Natl Acad Sci USA. 2015 Jun;112(22):6828-33.

Jiang S, Postovit L, Cattaneo A, Binder EB, Aitchison KJ. Epigenetic Modifications in Stress Response Genes Associated With Childhood Trauma. Front Psychiatry. 2019 Nov; 10:808.

Jones MJ, Islam SA, Edgar RD, Kobor MS. Adjusting for Cell Type Composition in DNA Methylation Data Using a Regression-Based Approach. Methods Mol Biol. 2017;1589:99106.

Jones PA. Functions of DNA methylation: islands, start sites, gene bodies and beyond. Nat Rev Genet. 2012 May;13(7):484-92.

Juster RP, McEwen BS, Lupien SJ. Allostatic load biomarkers of chronic stress and impact on health and cognition. Neurosci Biobehav Rev. 2010 Sep;35(1):2-16.

Kaliman P, Alvarez-López MJ, Cosín-Tomás M, Rosenkranz MA, Lutz A, Davidson RJ. Rapid changes in histone deacetylases and inflammatory gene expression in expert meditators. Psychoneuroendocrinology. 2014 Feb;40:96107.

Kaliman P. Epigenetics and meditation. Curr Opin Psychol. 2019 Aug;28:76-80.

Karg K, Burmeister M, Shedden K, Sen S. The serotonin transporter promoter variant (5-HTTLPR), stress, and depression meta-analysis revisited: evidence of genetic moderation. Arch Gen Psychiatry. 2011 May;68(5):44454.

Kurdyukov S, Bullock M. DNA Methylation Analysis: Choosing the Right Method. Biology (Basel). 2016 Jan;5(1):E3.

LaPlant Q, Vialou V, Covington HE 3rd, Dumitriu D, Feng J, Warren BL, et al. Dnmt3a regulates emotional behavior and spine plasticity in the nucleus accumbens. Nat Neurosci. 2010 Sep;13(9):1137-43.

Lester BM, Tronick E, Nestler E, Abel T, Kosofsky B, Kuzawa CW, et al. Behavioral epigenetics. Ann N Y Acad Sci. 2011 May;1226(1):14-33.

Lian Y, Xiao J, Wang Q, Ning L, Guan S, Ge H, et al. The relationship between glucocorticoid receptor polymorphisms, stressful life events, social support, and post-traumatic stress disorder. BMC Psychiatry. 2014 Aug;14(1):232.

Mason JW. A re-evaluation of the concept of "non-specificity" in stress theory. J Psychiatr Res. 1971 Aug;8(3):323-33.

McBride CM, Koehly LM. Imagining roles for epigenetics in health promotion research. J Behav Med. 2017 Apr;40(2):229-38.

McEwen BS, Wingfield JC. The concept of allostasis in biology and biomedicine. Horm Behav. 2003 Jan;43(1):2-15.

McEwen BS. In pursuit of resilience: stress, epigenetics, and brain plasticity. Ann N Y Acad Sci. 2016 Jun;1373(1):56-64.
Michels KB, Binder AM, Dedeurwaerder S, Epstein CB, Greally JM, Gut I, et al. Recommendations for the design and analysis of epigenome-wide association studies. Nat Methods. 2013 Oct;10(10):949-55.

Mifsud KR, Saunderson EA, Spiers H, Carter SD, Trollope AF, Mill J, et al. Rapid Down-Regulation of Glucocorticoid Receptor Gene Expression in the Dentate Gyrus after Acute Stress in vivo: Role of DNA Methylation and MicroRNA Activity. Neuroendocrinology. 2017;104(2):157-69.

Miller CA, Sweatt JD. Covalent modification of DNA regulates memory formation. Neuron. 2007 Mar;53(6):857-69.

Nikolova YS, Hariri AR. Can we observe epigenetic effects on human brain function? Trends Cogn Sci. 2015 Jul;19(7):366-73.

Oliveira AM, Hemstedt TJ, Bading H. Rescue of aging-associated decline in Dnmt3a2 expression restores cognitive abilities. Nat Neurosci. 2012 Jul;15(8):1111-3.

Palma-Gudiel H, Fañanás L. An integrative review of methylation at the serotonin transporter gene and its dialogue with environmental risk factors, psychopathology and 5-HTTLPR. Neurosci Biobehav Rev. 2017 Jan;72:190-209.

Park C, Rosenblat JD, Brietzke E, Pan Z, Lee Y, Cao B, et al. Stress, epigenetics and depression: A systematic review. Neurosci Biobehav Rev. 2019 Jul;102:139-52.

Pérez RF, Santamarina P, Tejedor JR, Urdinguio RG, Álvarez-Pitti J, Redon P, et al. Longitudinal genome-wide DNA methylation analysis uncovers persistent early-life DNA methylation changes. J Transl Med. 2019 Jan;17(1): 15.

Perroud N, Salzmann A, Prada P, Nicastro R, Hoeppli ME, Furrer S, et al. Response to psychotherapy in borderline personality disorder and methylation status of the BDNF gene. Transl Psychiatry. 2013 Jan; 3(1):e207.

Portela A, Esteller M. Epigenetic modifications and human disease. Nat Biotechnol. 2010 Oct; 28(10):1057-68.

Provenzi L, Guida E, Montirosso R. Preterm behavioral epigenetics: A systematic review. Neurosci Biobehav Rev. 2018 Jan;84:26271.

Rakyan VK, Down TA, Balding DJ, Beck S. Epigenome-wide association studies for common human diseases. Nat Rev Genet. 2011 Jul;12(8):529-41.

Roberts S, Keers R, Breen G, Coleman JR, Jöhren P, Kepa A, et al. DNA methylation of FKBP5 and response to exposure-based psychological therapy. Am J Med Genet B Neuropsychiatr Genet. 2019 Mar;180(2): 150-8.

Roberts S, Keers R, Lester KJ, Coleman JR, Breen $\mathrm{G}$, Arendt K, et al. HPA axis related genes and response to psychological therapies: genetics and epigenetics. Depress Anxiety. 2015 Dec; 32(12):861-70.

Roberts S, Lester KJ, Hudson JL, Rapee RM, Creswell C, Cooper PJ, et al. Serotonin transporter [corrected] methylation and response to cognitive behaviour therapy in children with anxiety disorders. Transl Psychiatry. 2014 Sep;4(9):e444. 
Rodrigues GM Jr, Toffoli LV, Manfredo $\mathrm{MH}$, Francis-Oliveira J, Silva AS, Raquel HA, et al. Acute stress affects the global DNA methylation profile in rat brain: modulation by physical exercise. Behav Brain Res. 2015 Feb;279: 123-8.

Sapolsky RM, Romero LM, Munck AU. How do glucocorticoids influence stress responses? Integrating permissive, suppressive, stimulatory, and preparative actions. Endocr Rev. 2000 Feb;21(1):55-89.

Schroeder M, Hillemacher T, Bleich S, Frieling H. The epigenetic code in depression: implications for treatment. Clin Pharmacol Ther. 2012 Feb;91(2):310-4.

Schuebel K, Gitik M, Domschke K, Goldman D. Making Sense of Epigenetics. Int J Neuropsychopharmacol. 2016 Dec;19(11):1-10.

Segerstrom SC, Miller GE. Psychological stress and the human immune system: a meta-analytic study of 30 years of inquiry. Psychol Bull. 2004 Jul;130(4):601-30.

Selye H. Stress in health and disease. Boston/London. Butterworth-Heinemann; 2013.

Shabalin AA, Aberg KA, van den Oord EJ. Candidate gene methylation studies are at high risk of erroneous conclusions. Epigenomics. 2015; $7(1): 13-5$.

Siegmund KD, Connor CM, Campan M, Long TI, Weisenberger DJ, Biniszkiewicz D, et al. DNA methylation in the human cerebral cortex is dynamically regulated throughout the life span and involves differentiated neurons. PLoS One. 2007 Sep;2(9):e895.

Stoffel M, Aguilar-Raab C, Rahn S, Steinhilber B, Witt SH, Alexander N, et al. Effects of Mindfulness-Based Stress Prevention on Serotonin Transporter Gene Methylation. Psychother Psychosom. 2019a;88(5):317-9.

Stoffel M, Gardini E, Abbruzzese E, Moessner M, Ehlert U, Ditzen B. Association of FKBP5 intron 7 methylation with diurnal cortisol patterns in healthy subjects. Psychoneuroendocrinology. 2019b;107 Suppl:11.

Stoffel M, Gardini E, Ehlert U, Ditzen B. Alterations in DNA methylation of FKBP5 following a stress prevention program. Psychoneuroendocrinology. 2017;83 Suppl:45.
Sweatt JD. Experience-dependent epigenetic modifications in the central nervous system. Biol Psychiatry. 2009 Feb;65(3):191-7.

Szyf M, Tang YY, Hill KG, Musci R. The dynamic epigenome and its implications for behavioral interventions: a role for epigenetics to inform disorder prevention and health promotion. Transl Behav Med. 2016 Mar;6(1): $55-62$.

Tang YY, Hölzel BK, Posner MI. The neuroscience of mindfulness meditation. Nat Rev Neurosci. 2015 Apr;16(4):213-25.

Techniker Krankenkasse. Entspann dich, Deutschland. TK-Stressstudie 2016. Hamburg, Techniker Krankenkasse, 2016. https:// www.tk.de/resource/blob/2026630/9154e4c7 1766 c410dc859916aa798217/tk-stressstudie2016-data.pdf

Turecki G, Meaney MJ. Effects of the Social Environment and Stress on Glucocorticoid Receptor Gene Methylation: A Systematic Review. Biol Psychiatry. 2016 Jan;79(2):87-96.

Unternaehrer E, Luers P, Mill J, Dempster E, Meyer AH, Staehli S, et al. Dynamic changes in DNA methylation of stress-associated genes (OXTR, BDNF) after acute psychosocial stress. Transl Psychiatry. 2012 Aug;2(8):e150.

Unternaehrer E, Meinlschmidt G. Psychosocial stress and DNA methylation. In: Spengler D, Binder E, editors. Epigenetics and Neuroendocrinology. Clinical Focus on Psychiatry. Cham: Springer; 2016. pp. 227-61.

Vaiserman AM. Epigenetic programming by early-life stress: evidence from human populations. Dev Dyn. 2015 Mar;244(3):254-65.

Van Daele T, Hermans D, Van Audenhove C, Van den Bergh O. Stress reduction through psychoeducation: a meta- analytic review. Health Educ Behav. 2012 Aug;39(4):474-85.

Varley KE, Gertz J, Bowling KM, Parker SL, Reddy TE, Pauli-Behn F, et al. Dynamic DNA methylation across diverse human cell lines and tissues. Genome Res. 2013 Mar;23(3):555-67.

Varvogli L, Darviri C. Stress Management Techniques: evidence-based procedures that reduce stress and promote health. Health Sci J. 2011;5(2):74-89.

Weaver IC, Cervoni N, Champagne FA, D’Alessio AC, Sharma S, Seckl JR, et al. Epigenetic programming by maternal behavior. Nat Neurosci. 2004 Aug;7(8):847-54.
World Health Organization. Psychische Gesundheit: Herausforderungen annehmen, Lösungen schaffen: Bericht über die Ministerkonferenz der Europäischen Region der WHO. Kopenhagen, WHO-Regionalbüro für Europa, 2006. http://www.euro.who.int/_data/ assets/pdf_file/0009/96453/E87301G.pdf

Wray NR, Ripke S, Mattheisen M, Trzaskowski M, Byrne EM, Abdellaoui A, et al.; eQTLGen; 23andMe; Major Depressive Disorder Working Group of the Psychiatric Genomics Consortium. Genome-wide association analyses identify 44 risk variants and refine the genetic architecture of major depression. Nat Genet. 2018 May;50(5):668-81.

$\mathrm{Wu}$ Ct, Morris JR. Genes, genetics, and epigenetics: a correspondence. Science. 2001 Aug;293(5532):1103-5.

Yehuda R, Daskalakis NP, Desarnaud F, Makotkine I, Lehrner AL, Koch E, et al. Epigenetic Biomarkers as Predictors and Correlates of Symptom Improvement Following Psychotherapy in Combat Veterans with PTSD. Front Psychiatry. 2013 Sep;4:118.

Zannas AS, Binder EB. Gene-environment interactions at the FKBP5 locus: sensitive periods, mechanisms and pleiotropism. Genes Brain Behav. 2014 Jan;13(1):25-37.

Zannas AS, West AE. Epigenetics and the regulation of stress vulnerability and resilience. Neuroscience. 2014 Apr;264:157-70.

Zannas AS, Wiechmann T, Gassen NC, Binder EB. Gene-Stress-Epigenetic Regulation of FKBP5: Clinical and Translational Implications. Neuropsychopharmacology. 2016 Jan; 41(1):261-74

Zhang B, Zhou Y, Lin N, Lowdon RF, Hong C, Nagarajan RP, et al. Functional DNA methylation differences between tissues, cell types, and across individuals discovered using the M\&M algorithm. Genome Res. 2013 Sep; 23(9):1522-40.

Ziegler C, Dannlowski U, Bräuer D, Stevens S, Laeger I, Wittmann H, et al. Oxytocin receptor gene methylation: converging multilevel evidence for a role in social anxiety. Neuropsychopharmacology. 2015 May;40(6):152838. 